\title{
Use of strain-energy method for calculating rectangular beams in cases of lateral buckling
}

\author{
Aleksandr Ishchenko ${ }^{1 *}$ and Ivan Zotov ${ }^{2}$ \\ ${ }^{1}$ Moscow state university of civil engineering, Yaroslavskoye sh., 26, 129337, Moscow, Russia \\ ${ }^{2}$ Don state technical university, pl. Gagarina, 1, Rostov-on-Don, 344002, Russia
}

\begin{abstract}
The paper deals with the lateral buckling problem of a freely supported wooden strip with a constant narrow cross section, loaded with a local force in the middle of the span. A differential equation is given for cases when the force is applied out of the gravity section center. Strainenergy method was used in the study of beam lateral buckling. In the case when the load is applied in the center of gravity, problem comes down to a generalized characteristic equation. The correlation between the magnitude of the critical force and the application point of the load was obtained. The linear approximating function was identified for the indicated dependence. The obtained results are compared with an analytical solution using the Bessel functions and a numerical iterative method.
\end{abstract}

\section{Introduction}

It is known that a beam with a narrow rectangular cross-section, which bends in its plane under local load A applied in the gravity center of the middle cross section, can laterally buckle at a known load value (Fig. 1.).

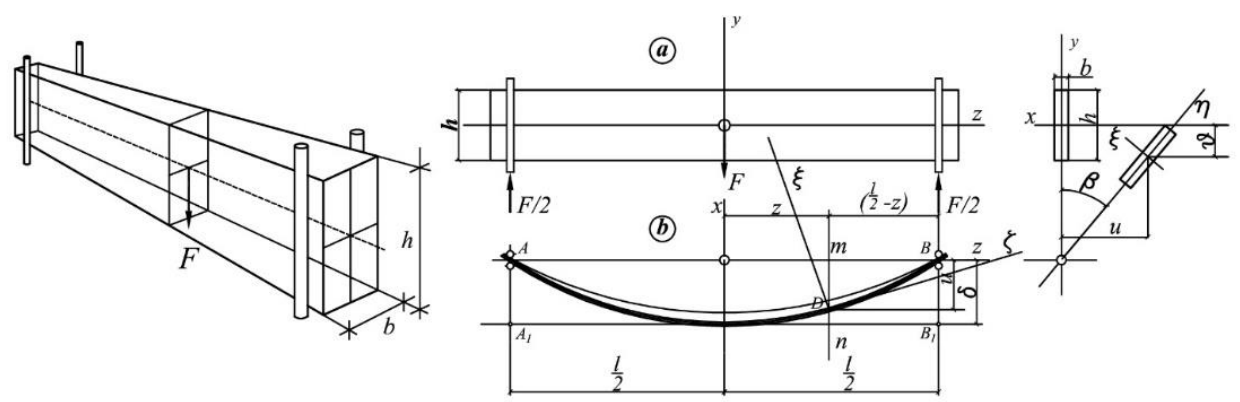

Fig. 1. The plain bending analytic model.

* Corresponding author: ibm2225101@gmail.com 


\section{Results}

An arbitrary section $n-m$ with coordinates $\xi, \eta, \zeta$ is considered. The deformation of the strip is determined by the two components of the displacement $u$ and $v$, as well as the angle $\beta$, by which it turns. The flection values of the strip central axes in the $X Z$ and $Y Z$ planes are shown in Fig. 1. They will be $\frac{\partial^{2} u}{d z^{2}}$ and $\frac{\partial^{2} v}{d z^{2}}$, respectively. It is possible to assume that for a small angle $\beta$ the flection in the planes $\xi, \eta, \zeta$ has the same values. The principal axis rotation angle per unit length will be $\frac{\partial \beta}{d z}$. Then the equilibrium equation has the following form $[4,6,7]$ :

$$
\left\{\begin{array}{c}
B_{1} \cdot \frac{\partial^{2} u}{d z^{2}}=M_{\eta} \\
B_{2} \cdot \frac{\partial^{2} v}{d z^{2}}=-M_{\xi} \\
\mathrm{C} \cdot \frac{\partial \beta}{d z}=M_{\zeta}
\end{array}\right.
$$

Here: $C=G \frac{b^{3} h}{3}\left(1-0,630 \frac{h}{b}\right), B_{1}=E \frac{b^{3} h}{12}, B_{2}=E \frac{h^{3} b}{12}$ are the torsion and binding stiffness, respectively. $M_{\eta}, M_{\xi}, M_{\zeta}-$ moments about the respective axes of forces applied to the strip part lying to the right of $m-n$ cross section.

It should be noted that the moments are considered positive when act in the same directions as were indicated for positive rotations about the axes $\xi, \eta$ and $\zeta$.

For the further derivation of resolving equations, expressions are necessary for the cosines of the angles between the coordinate axes $\xi, \eta, \zeta$ and $X, Y, Z$.

Assuming the displacement values of $u, v$ and $\beta$ are very small, the cosines required for the calculations will take the following values:

Table 1. The cosines values between the coordinate axes $\xi, \eta, \zeta$ and $X, Y, Z$.

\begin{tabular}{|c|c|c|c|}
\hline Coordinate axes & $X$ & $Y$ & $Z$ \\
\hline$\xi$ & 1 & $\beta$ & $-\frac{\partial u}{\partial z}$ \\
\hline$\eta$ & $-\beta$ & 1 & $-\frac{\partial v}{\partial z}$ \\
\hline$\zeta$ & $\frac{\partial u}{\partial z}$ & $\frac{\partial v}{\partial z}$ & 1 \\
\hline
\end{tabular}

When the equilibrium of the right side of the strips cross section $m-n$ is considered, it can be seen that the forces acting on this part come down to the vertical force $\frac{F}{2}$ and the reactive torque $\frac{F}{2} \cdot \delta$ acting at point $\mathrm{B}$.

Moments about the axes with a beginning at the $\mathrm{D}$ point center of the cross section and parallel to the axes $X, Y$ and $Z$ will be as follows:

$$
M_{x}=-\frac{F}{2}\left(\frac{l}{2}-z\right) ; \quad M_{y}=0 ; \quad M_{z}=\frac{F}{2}(\delta-u)
$$

where $\delta$ is lateral deflection in the middle of the beam, and $u$ is deflection in the $m-n$ cross section.

Using the cosine table (Fig.2) the following can be obtained: 


$$
\left\{\begin{array}{l}
M_{\xi}=-\frac{F}{2}\left(\frac{l}{2}-z\right)-\frac{F}{2}(\delta-u) \frac{\partial u}{\partial z} \\
M_{\eta}=\frac{F}{2}\left(\frac{l}{2}-z\right) \beta-\frac{F}{2}(\delta-u) \frac{\partial v}{\partial z} \\
M_{\zeta}=-\frac{F}{2}\left(\frac{l}{2}-z\right) \frac{\partial u}{\partial z}-\frac{F}{2}(\delta-u)
\end{array}\right.
$$

After substituting expression (2) into (1), the following differential equations can be obtained:

$$
\left\{\begin{array}{c}
B_{1} \cdot \frac{\partial^{2} u}{d z^{2}}=\frac{F}{2}\left(\frac{l}{2}-z\right) \beta-\frac{F}{2}(\delta-u) \frac{\partial v}{\partial z} \\
B_{2} \cdot \frac{\partial^{2} v}{d z^{2}}=\frac{F}{2}\left(\frac{l}{2}-z\right)+\frac{F}{2}(\delta-u) \frac{\partial u}{\partial z} \\
\mathrm{C} \cdot \frac{\partial \beta}{d z}=-\frac{F}{2}\left(\frac{l}{2}-z\right) \frac{\partial u}{\partial z}-\frac{F}{2}(\delta-u)
\end{array}\right.
$$

After eliminating the displacement $u$ from the first and third equations, and assuming that $v=0$ due to its smallness, it can be obtained:

$$
\frac{\partial^{2} \beta}{d z^{2}}+\frac{F^{2}}{4 B_{1} \mathrm{C}}\left(\frac{l}{2}-z\right)^{2} \cdot \beta=0
$$

A numerical solution in a finite-difference model and a solution in infinite series gives the result of the critical force $[3,10]$ :

$$
F_{c r}=\frac{16,93 \sqrt{B_{1} \mathrm{C}}}{l^{2}} ;
$$

In many cases, it is possible to use the energy method with great advantage for the study of beam lateral buckling. In order to demonstrate the use of this method, the case presented in Fig. 1 should be considered again.

When the beam buckles to the side, the deformation energy of the beam increases, since the bending of the beam in the load application plane is accompanied by a bend in the transverse direction and torsion about the longitudinal axis. At the same time, the load application point decreases and the load does some work. The critical load value is now determined according to the condition that this work is equal to the lateral bending deformation energy combined with the torsional deformation energy. In this case, we can neglect a small change in the beam bend energy in its plane, which occurs during buckling. This equals the previous assumption made when creating differential equilibrium equations, which stated that the plane flection of the wall is infinitely small and can be neglected. The result obtained using this way is quite accurate when the stiffness of the beam in the wall plane is infinitely large, and it is fairly correct if this stiffness is very large compared to the stiffness in the transverse direction, which is the usual practical example.

Conventional formulas can be used in order to determine the strain energy of bending and torsion. In this case, taking into account the symmetry of the bulking beam shape (Fig. 1), an increase in the deformation energy due to buckling can be obtained in the following form:

$$
E=B_{1} \cdot \int_{0}^{0,5 l}\left(\frac{\partial^{2} u}{\partial z^{2}}\right)^{2} d z+C \cdot \int_{0}^{0,5 l}\left(\frac{\partial \beta}{\partial z}\right)^{2} d z
$$


In order to determine the $F$ load pulldown during lateral buckling, the element of the beam longitudinal axis at the point D (Fig. 1) should be considered. If the cross section $m-n$ is considered as fixed, then due to the plane bending of this element the beam end $\mathrm{B}$ will describe an infinitely small arc in the plane:

$$
\frac{\partial^{2} u}{\partial z^{2}}\left(\frac{l}{2}-z\right) d z
$$

vertical component of which equals the following:

$$
\beta \frac{\partial^{2} u}{\partial z^{2}}\left(\frac{l}{2}-z\right) d z
$$

By changing $\mathrm{z}$ from 0 to $l / 2$ and summing up the vertical components stated in (8), the decrease of the load point $\mathrm{F}$ can be obtained due to lateral buckling of the beam as an integral:

$$
\int_{0}^{0,5 l} \beta \frac{\partial^{2} u}{\partial z^{2}}\left(\frac{l}{2}-z\right) d z
$$

The equation for determining the critical load becomes as follows:

$$
F \cdot \int_{0}^{0,5 l} \beta \frac{\partial^{2} u}{\partial z^{2}}\left(\frac{l}{2}-z\right) d z=B_{1} \cdot \int_{0}^{0,5 l}\left(\frac{\partial^{2} u}{\partial z^{2}}\right)^{2} d z+C \cdot \int_{0}^{0,5 l}\left(\frac{\partial \beta}{\partial z}\right)^{2} d z
$$

After substituting the value, which follows from the first equation (3) in place of $\frac{\partial^{2} u}{\partial z^{2}}$ :

$$
\frac{F^{2}}{4 B_{1}} \cdot \int_{0}^{0,5 l} \beta^{2}\left(\frac{l}{2}-z\right)^{2} d z=C \cdot \int_{0}^{0,5 l}\left(\frac{\partial \beta}{\partial z}\right)^{2} d z
$$

In order to determine the critical load $F$ value, it is necessary to adopt a suitable analytical expression for the torsion angle $\beta$ that satisfies the conditions at the ends of the beam, and substitute it into equation (10). The general expression for $\beta$, which satisfies the conditions at the ends, can be adopted in the form of a trigonometric series:

$$
\beta=a_{1} \cos \frac{\pi z}{l}+a_{2} \cos \frac{3 \pi z}{l}+a_{3} \cos \frac{5 \pi z}{l}+\cdots
$$

Using only the first term of this series for $\beta$ as the first approximation, then substituting it into equation (10) and integrating, the following can be obtained:

$$
F_{c r}=\frac{17,2 \sqrt{B_{1} C}}{l^{2}}
$$

The numerical solution of the finite difference model and the solution obtained by using the energy method gives a $1.5 \%$ difference in the results of the crippling load. If the first two terms of the series (11) are taken as the second approximation and the constants $a_{1}$ and $a_{2}$ are adopted to make minimal $F_{c r}$, the error will be less than $0.1 \%$.

To sum up, the last example shows that the energy method can be successfully applied for studying lateral buckling of beams. Over and above, in the case of using the energy method the complex integration of differential equations by the method of infinite series is replaced by calculation of simple integrals included in equation (10), and a relatively simple expression for $\beta$ usually allows to obtain $F_{c r}$ with an accuracy sufficient for 
practical purposes. Usually $F_{c r}$ determined by the energy method is always higher than its true value.

The value of the crippling load $F_{c r}$ depends on its application point. It is obvious that the load application above the center of the cross section reduces its critical value, and application below the center produces the opposite effect. The power of this effect is easy to obtain by using the energy method; it is only necessary to take into account the additional pulldown of the load $F$ due to lateral buckling of the beam caused by the rotation of the middle cross section. If we take $\beta_{0}-$ the rotation angle and $a-$ the vertical distance between the load application point and the cross section center (positive when it is at the top), then the additional load decrease will be:

$$
a \cdot\left(1-\cos \beta_{0}\right) \approx \frac{a \beta_{0}^{2}}{2}
$$

Then instead of the equation (10) the following can be obtained:

$$
F \frac{a \beta_{0}^{2}}{2}+\frac{F^{2}}{4 B_{1}} \cdot \int_{0}^{0,5 l} \beta^{2}\left(\frac{l}{2}-z\right)^{2} d z=C \cdot \int_{0}^{0,5 l}\left(\frac{\partial \beta}{\partial z}\right)^{2} d z
$$

If $a \rightarrow 0$, then the first left side member of (14) is small and can be substituted by the value (5) for $F$. Thus, the following approximate formula is obtained:

$$
F_{c r}=\frac{16,93 \sqrt{B_{1} C}}{l^{2}} \cdot\left(1-\frac{3,48 a}{l} \sqrt{\frac{B_{1}}{C}}\right)
$$

\section{Conclusion}

A comparison was made with the results obtained by using the energy method with the iteration algorithm and the analytical solution of A.S. Volmira based on Bessel functions (Table 2).

Comparison of the results obtained by the means of iterative algorithm and an analytical

\begin{tabular}{|c|c|c|c|c|c|c|c|}
\hline \multicolumn{8}{|c|}{ Above the gravity center } \\
\hline$a$ & 0 & \multicolumn{2}{|l|}{0.03} & 0.143 & \multicolumn{2}{|l|}{0.293} & 0.544 \\
\hline $\begin{array}{l}\mathrm{K} \\
\text { (Chepurnenko) }\end{array}$ & 16.94 & \multicolumn{2}{|c|}{15.9857} & 12.7765 & \multicolumn{2}{|l|}{9.6056} & 6.3512 \\
\hline K (Volmir) & 16.94 & \multicolumn{2}{|l|}{16.0} & 12.8 & \multicolumn{2}{|l|}{\begin{tabular}{|l|}
9.6 \\
\end{tabular}} & 6.4 \\
\hline $\mathrm{K}$ (authors) & 16.93 & 15.96 & & 12.7265 & 9.5956 & & 6.3498 \\
\hline \multicolumn{8}{|c|}{ Below the gravity center } \\
\hline & 0 & -0.069 & -0.166 & -0.271 & -0.396 & -0.562 & -0.815 \\
\hline $\begin{array}{l}\mathrm{K} \\
\text { (Chepurnenko) }\end{array}$ & 16.94 & 19.2151 & 22.3576 & 25.5395 & 28.7354 & 31.9455 & 35.1239 \\
\hline K (Volmir) & 16.94 & 19.2 & 22.4 & 25.6 & 28.8 & 32.0 & 35.2 \\
\hline K (authors) & 16.93 & 19.2091 & 22.378 & 25.5475 & 28.7732 & 31.9832 & 35.1443 \\
\hline
\end{tabular}
solution of A.S. Volmira [10] based on the Bessel functions was made (Table 2).

Table 2. Bessel functions.

The coincidence of the results indicates their reliability. 


\section{References}

1. A.A. Karamysheva, B.M. Yazyyev, A.S. Chepurnenko, S.B. Yazyyeva, Inzhenernyy vestnik Dona 3, 1-7 (2015)

2. A.A. Karamysheva, B.M. YAzyyev, A.S. Chepurnenko, S.B. Yazyyeva Inzhenernyy vestnik Dona 3, 8-15 (2015)

3. A.S. Chepurnenko, V.V. Ul'yanskaya, D.A. Vysokovskiy, I.M. Zotov, Inzhenernyy vestnik Dona 3, 16-26 (2015)

4. S.P. Timoshenko, J.M. Gere, Theory of elastic stability (New York, McGraw-Hill, 1961)

5. A.A. Karamysheva, S.B. Yazyev, A.A. Avakov, ICIE 1, 1872-1877 (2016)

6. A.S. Vol'mir, Ustoychivost' deformiruyemykh system (Nauka, Moscow, 1967)

7. S.P. Timoshenko, Ustoychivost' uprugikh system (Gostekhizdat, Moscow, 1946)

8. P.M. Varvak, L.P. Varvak, Metod setok v zadachakh rascheta stroitel'nykh konstruktsiy (Stroyizdat, Moscow, 1977)

9. S. Micu, I. Rovenţa, L.E. Temereancă, Approximation of the controls for the linear beam equation (Springer-Verlag, London, 2016) 